\title{
Stochastic Differential Equations with Reflecting Boundary Condition in Convex Regions
}

\author{
Hiroshi TaNaka \\ (Received September 16, 1978)
}

\section{§1. Introduction}

A. V. Skorohod [4] considered a stochastic differential equation for a reflecting diffusion process on $\bar{D}=[0, \infty$ ) (see also McKean [2] [3]). This is the simplest case among stochastic differential equations subject to boundary conditions and can be solved easily. The purpose of this paper is to show that the multi-dimensional version of Skorohod's equation is still easy to solve if we assume that the domain $D$ is convex.

Skorohod's equation describing a reflecting Brownian path $\xi$ on $\bar{D}=[0, \infty)$ is

$$
\xi=w+\varphi,
$$

where $w$ is a standard Brownian path and $\xi$ is to be found as a $\bar{D}$-valued continuous function under the condition that $\varphi(t)$ increases only when $\xi(t)=0$. The equation (1.1) has a unique solution not only for almost all Brownian paths but also for all continuous functions $w$ with $w(0) \in \bar{D}$, and the solution is given by

$$
\xi(t)= \begin{cases}w(t) & \text { for } 0 \leq t \leq T, \\ w(t)-\inf \{w(s): T \leq s \leq t\} & \text { for } t>T,\end{cases}
$$

where $T=\inf \{t>0: w(t)<0\}$. Our first problem is to consider a multi-dimensional version of the equation (1.1) assuming that $D$ is a convex domain. Although we can not obtain an explicit formula for the solution like (1.2), we are able to construct the unique solution $\xi$ for any $\mathbf{R}^{d}$-valued continuous function $w$ with $w(0) \in \bar{D}$ and to prove that $\xi$ depends continuously on $w$, if $D$ is a convex domain in $\mathbf{R}^{d}$ satisfying certain condition (Theorem 2.1). This additional condition is automatically satisfied if $D$ is bounded or $d=2$. This result will lead to a simple solution to our second problem which is concerned with a stochastic differential equation with (normal) reflection having variable coefficients similar to Skorohod's. The following may be stressed.

(i) The boundary does not need to be smooth as far as the domain is assumed to be convex.

(ii) The diffusion coefficients may degenerate (however, in this case the path of 
the solution might not behave like an ordinary reflection).

Our results are roughly stated as follows. The convexity assumption for the domain makes the situation quite similar to that in the whole space; in fact, the existence of solutions will be obtained assuming only the bounded continuity of the coefficients (Theorem 4.2), and the pathwise uniqueness of solutions will be proved under the same regularity assumption on the coefficients as found in the work of Watanabe and Yamada [9] for the case of whole space (Theorem 4.3).

However, it is noted that our methods and results are restricted to the case of reflecting boundary condition; the convexity assumption will not simplify the situation in the case of general boundary conditions such as discussed by Ikeda [1], Watanabe [8], Stroock and Varadhan [6] and Tsuchiya [7].

\section{§2. A deterministic problem}

An $\mathbf{R}^{d}$-valued function $\varphi(t)=\left(\varphi^{1}(t), \ldots, \varphi^{d}(t)\right)$ defined on $\mathbf{R}_{+}=[0, \infty)$ is said to be of bounded variation for simplicity if the component functions are of bounded variation on each finite $t$-interval. Given such a function $\varphi(t)$ which is right continuous and $\varphi(0)=0$, we put

$$
\begin{aligned}
|\varphi|(t) & =\text { the total variation of } \varphi \text { on }[0, t] \\
& =\sup \sum_{k}\left|\varphi\left(t_{k}\right)-\varphi\left(t_{k-1}\right)\right|,
\end{aligned}
$$

where the supremum is taken over all partitions: $0=t_{0}<t_{1}<\cdots<t_{n}=t . \quad \varphi(t)$ can be expressed as

$$
\varphi(t)=\int_{0}^{t} \boldsymbol{n}(s) d|\varphi|(s)=\int_{[0, t]} \boldsymbol{n}(s)|\varphi|(d s)
$$

with a unit vector valued function $\boldsymbol{n}(t) ; \boldsymbol{n}(t)$ is uniquely determined almost everywhere with respect to the measure $d|\varphi|$.

Let $D$ be a convex domain in $\mathbf{R}^{d}$ and $\bar{D}$ its closure; $D$ will be fixed throughout. For $x \in \partial D$ we denote by $\mathscr{H}_{x}(D)$ the set of all supporting hyperplanes of $D$ at $x$. By (an inward) normal vector at $x \in \partial D$ we mean any inward unit vector perpendicular to some $H \in \mathscr{H}_{x}(D)$, and denote by $\mathscr{N}_{x}(D)$ the set of all inward normal vectors at $x \in \partial D$. Of course, it can happen that $\# \mathscr{N}_{x}(D)=\infty$ unless $\partial D$ is smooth near $x$. We shall also consider the following spaces of functions.

$\mathbf{C}\left(\mathbf{R}_{+}, \mathbf{R}^{d}\right) \quad\left(\operatorname{resp} . \mathbf{C}\left(\mathbf{R}_{+}, \bar{D}\right)\right)=$ the space of $\mathbf{R}^{d}$-valued (resp. $\bar{D}$-valued) continuous functions on $\mathbf{R}_{+}$.

$\mathbf{D}\left(\mathbf{R}_{+}, \mathbf{R}^{d}\right)$ (resp. $\left.\mathbf{D}\left(\mathbf{R}_{+}, \bar{D}\right)\right)=$ the space of $\mathbf{R}^{d}$-valued (resp. $\bar{D}$-valued) right continuous functions on $\mathbf{R}_{+}$with left limits. 
On $\mathbf{C}\left(\mathbf{R}_{+}, \mathbf{R}^{d}\right)$ and $\mathbf{C}\left(\mathbf{R}_{+}, \bar{D}\right)$ we consider the compact uniform topology. Given a function $\xi$ in $\mathbf{D}\left(\mathbf{R}_{+}, \bar{D}\right)$, a function $\varphi$ is said to be associated with $\xi$ if the following three conditions are satisfied.

(2.2) $\varphi$ is a function in $\mathbf{D}\left(\mathbf{R}_{+}, \mathbf{R}^{d}\right)$ with bounded variation and $\varphi(0)=0$.

(2.3) The set $\left\{t \in \mathbf{R}_{+}: \xi(t) \in D\right\}$ has $d|\varphi|$-measure zero.

(2.4) The function $\boldsymbol{n}(t)$ appearing in the expression (2.1) is a normal vector at $\xi(t)$ for almost all $t$ with respect to the measure $d|\varphi|$.

REMARK 2.1. The condition (2.4) can be replaced by the following one.

(2.4') For any $\eta \in \mathbf{C}\left(\mathbf{R}_{+}, \bar{D}\right),(\eta(t)-\xi(t), \varphi(d t)) \geq 0$.

Example. Let $\partial D$ be smooth, $\boldsymbol{n}(x)$ the inward normal vector at $x \in \partial D$ and $\xi \in \mathbf{D}\left(\mathbf{R}_{+}, \bar{D}\right)$. Then, for any right continuous non-decreasing function $\rho(t)$ on $\mathbf{R}_{+}$with $\rho(0)=0$

$$
\varphi(t)=\int_{0}^{t} \mathbf{1}_{\partial D}(\xi(s)) \boldsymbol{n}(\xi(s)) d \rho(s)
$$

is clearly an associated function of $\xi$.

Our first problem stated in the introduction can now be formulated as follows.

Problem. Given $w \in \mathbf{D}\left(\mathbf{R}_{+}, \mathbf{R}^{d}\right)$ with $w(0) \in \bar{D}$, find a solution $\xi$ of

$$
\xi=w+\varphi .
$$

When we speak of the equation (2.5), it is always understood that $\xi \in \mathbf{D}\left(\mathbf{R}_{+}, \bar{D}\right)$ and $\varphi$ is associated with $\xi$.

As stated in the introduction, in the simplest case $\bar{D}=[0, \infty)$ the solution of (2.5) is given by (1.2). However, in the general multi-dimensional case the existence of a solution of (2.5) is not trivial. An example in which a solution of (2.5) can easily be found is the case when $w$ is a step function as will be seen in the lemma below. For a given point $x \in \mathbf{R}^{d}-\bar{D}$ we denote by $[x]_{0}$ the (unique) point on $\partial D$ which gives the minimum distance between $x$ and $\bar{D}$.

Lemma 2.1. If $w$ is a step function with $w(0) \in \bar{D}$, then a solution of (2.5) exists.

Proof. Put $T_{1}=\inf \{t>0: w(t) \notin \bar{D}\}$ and define $\xi(t), 0 \leq t \leq T_{1}$, by $\xi(t)$ $=w(t)$ for $t<T_{1}$ and $\xi\left(T_{1}\right)=\left[w\left(T_{1}\right)\right]_{\partial}$. Then, $T_{1}>0$ and $\xi(t)$ solves (2.5) for $0 \leq t \leq T_{1}$. Next, suppose a solution $\xi(t)$ of (2.5) is obtained for $0 \leq t \leq T_{n-1}$ and put 


$$
\begin{aligned}
& T_{n}=\inf \left\{t>T_{n-1}: w(t)+\varphi\left(T_{n-1}\right) \notin \bar{D}\right\}, \\
& \xi(t)= \begin{cases}w(t)+\varphi\left(T_{n-1}\right) & \text { for } T_{n-1}<t<T_{n}, \\
{\left[w\left(T_{n}\right)+\varphi\left(T_{n-1}\right)\right]_{\diamond}} & \text { for } t=T_{n} .\end{cases}
\end{aligned}
$$

Then, $\xi(t)$ solves (2.5) for $0 \leq t \leq T_{n}$. Repeating this argument, we can obtain a solution of (2.5) for $0 \leq t<\infty$ because $T_{n} \uparrow \infty$ as $n \uparrow \infty$.

Lemma 2.2. (i) Let $w, \tilde{w} \in \mathbf{D}\left(\mathbf{R}_{+}, \mathbf{R}^{d}\right)$ with $w(0), \tilde{w}(0) \in \bar{D}$, and $\xi, \tilde{\xi}$ be any solutions of

$$
\xi=w+\varphi, \quad \tilde{\xi}=\tilde{w}+\tilde{\varphi},
$$

respectively. Then we have

$$
\begin{aligned}
|\xi(t)-\tilde{\xi}(t)|^{2} \leq & |w(t)-\tilde{w}(t)|^{2} \\
& +2 \int_{0}^{t}(w(t)-\tilde{w}(t)-w(s)+\tilde{w}(s), \varphi(d s)-\tilde{\varphi}(d s)) .
\end{aligned}
$$

(ii) If $\xi$ is a solution of (2.5), then

$$
\begin{aligned}
|\xi(t)-\xi(s)|^{2} \leq & |w(t)-w(s)|^{2} \\
& +2 \int_{(s, t]}(w(t)-w(\tau), \varphi(d \tau)), \quad 0 \leq s \leq t .
\end{aligned}
$$

Proof. (i) We have

$$
\begin{aligned}
& |\varphi(t)-\tilde{\varphi}(t)|^{2}=\int_{0}^{t} \int_{0}^{t}\left(\varphi\left(d t_{1}\right)-\tilde{\varphi}\left(d t_{1}\right), \varphi\left(d t_{2}\right)-\tilde{\varphi}\left(d t_{2}\right)\right) \\
& =2 \iint_{0 \leq t_{1} \leq t_{2} \leq t}\left(\varphi\left(d t_{1}\right)-\tilde{\varphi}\left(d t_{1}\right), \varphi\left(d t_{2}\right)-\tilde{\varphi}\left(d t_{2}\right)\right) \\
& -\sum_{0 \leq s \leq t}|\varphi(s)-\tilde{\varphi}(s)-\varphi(s-)+\tilde{\varphi}(s-)|^{2} \\
& \leq 2 \int_{0}^{t}(\varphi(s)-\tilde{\varphi}(s), \varphi(d s)-\tilde{\varphi}(d s)), \\
& (w(t)-\tilde{w}(t), \varphi(t)-\tilde{\varphi}(t))=\int_{0}^{t}(w(t)-\tilde{w}(t), \varphi(d s)-\tilde{\varphi}(d s)) \\
& =\int_{0}^{t}(w(t)-\tilde{w}(t)-w(s)+\tilde{w}(s), \varphi(d s)-\tilde{\varphi}(d s)) \\
& +\int_{0}^{t}(w(s)-\tilde{w}(s), \varphi(d s)-\tilde{\varphi}(d s)) .
\end{aligned}
$$

Therefore 


$$
\begin{aligned}
|\xi(t)-\tilde{\xi}(t)|^{2}= & |w(t)-\tilde{w}(t)|^{2}+2(w(t)-\tilde{w}(t), \varphi(t)-\tilde{\varphi}(t)) \\
& +|\varphi(t)-\tilde{\varphi}(t)|^{2} \\
\leq & |w(t)-\tilde{w}(t)|^{2}+2 \int_{0}^{t}(\xi(s)-\tilde{\xi}(s), \varphi(d s)-\tilde{\varphi}(d s)) \\
& +2 \int_{0}^{t}(w(t)-\tilde{w}(t)-w(s)+\tilde{w}(s), \varphi(d s)-\tilde{\varphi}(d s)) .
\end{aligned}
$$

But the second term is non-positive by $\left(2.4^{\prime}\right)$ of Remark 2.1 .

(ii) By a method similar to the case (i), we have

$$
\begin{aligned}
|\xi(t)-\xi(s)|^{2}= & |w(t)-w(s)|^{2}+|\varphi(t)-\varphi(s)|^{2} \\
& +2(w(t)-w(s), \varphi(t)-\varphi(s)) \\
\leq & |w(t)-w(s)|^{2}+2 \int_{(s, t]}(\xi(\tau)-\xi(s), \varphi(d \tau)) \\
& +2 \int_{(s, t]}(w(t)-w(\tau), \varphi(d \tau))
\end{aligned}
$$

But the second term is non-positive by $\left(2.4^{\prime}\right)$; the proof is finished.

REMARK 2.2. By a method similar to the above, we can prove the following: If $w$ and $\tilde{w}$ of (i) of Lemma 2.2 are replaced by $w+a$ and $\tilde{w}+\tilde{a}$, respectively, where $a$ and $\tilde{a}$ are $\mathbf{R}^{d}$-valued right continuous functions of bounded variation with $a(0)=\tilde{a}(0)=0$, then

$$
\begin{aligned}
\mid \xi(t) & -\left.\tilde{\xi}(t)\right|^{2} \leq|w(t)-\tilde{w}(t)|^{2}+2 \int_{0}^{t}(\xi(s)-\tilde{\xi}(s), a(d s)-\tilde{a}(d s)) \\
& +2 \int_{0}^{t}(w(t)-\tilde{w}(t)-w(s)+\tilde{w}(s), a(d s)+\varphi(d s)-\tilde{a}(d s)-\tilde{\varphi}(d s)) .
\end{aligned}
$$

By a similar replacement of $w$ in (ii) by $w+a$, the inequality in (ii) becomes

$$
\begin{aligned}
\mid \xi(t) & -\left.\xi(s)\right|^{2} \leq|w(t)-w(s)|^{2}+2 \int_{(s, t]}(\xi(\tau)-\xi(s), a(d \tau)) \\
& +2 \int_{(s, t]}(w(t)-w(\tau), a(d \tau)+\varphi(d \tau)) .
\end{aligned}
$$

Lемма 2.3. (2.5) has at most one solution.

Proof. Let $\xi$ and $\tilde{\xi}$ be solutions of (2.5). Then, putting $w=\tilde{w}$ in (i) of Lemma 2.2 we obtain $|\xi(t)-\tilde{\xi}(t)|^{2} \leq 0$.

Lemma 2.4. If $w$ is continuous, then the solution of (2.5) is also continuous. 
Proof. From (ii) of Lemma 2.2 we have

$$
|\xi(t)-\xi(s)|^{2} \leq|w(t)-w(s)|^{2}+2 \int_{(s, t]}|w(t)-w(\tau)||\varphi|(d \tau),
$$

from which the continuity of $\xi$ follows.

Lemмa 2.5. Let $\left\{w_{n}\right\}_{n \geq 1}$ be a sequence in $\mathbf{D}\left(\mathbf{R}_{+}, \mathbf{R}^{d}\right)$ such that for each $n$ the equation $\xi_{n}=w_{n}+\varphi_{n}$ has a solution for $0 \leq t \leq T$, $T$ being a positive constant. If $w_{n}$ converges uniformly on $[0, T]$ to some $w \in \mathbf{C}\left(\mathbf{R}_{+}, \mathbf{R}^{d}\right)$ as $n \rightarrow \infty$ and if $\left\{\left|\varphi_{n}\right|(T)\right\}_{n \geq 1}$ is bounded, then $\xi_{n}$ converges uniformly on $[0, T]$ as $n \rightarrow \infty$ to the solution $\xi$ of $\xi=w+\varphi$ for $0 \leq t \leq T$. we have

Proof. Let $K$ be the bound of $\left\{\left|\varphi_{n}\right|(T)\right\}_{n \geq 1}$. Then applying Lemma 2.2,

$$
\begin{gathered}
\left|\xi_{n}(t)-\xi_{m}(t)\right|^{2} \leq\left|w_{n}(t)-w_{m}(t)\right|^{2}+8 K \sup _{0 \leq s \leq t}\left|w_{n}(s)-w_{m}(s)\right|, \\
\left|\xi_{n}(t)-\xi_{n}(s)\right|^{2} \leq\left|w_{n}(t)-w_{n}(s)\right|^{2}+2 K \sup _{s \leq t_{1} \leq t_{2} \leq t}\left|w_{n}\left(t_{2}\right)-w_{n}\left(t_{1}\right)\right| .
\end{gathered}
$$

From the first inequality it follows that $\left\{\xi_{n}\right\}_{n \geq 1}$ is uniformly convergent on $[0, T]$ and hence the same for $\left\{\varphi_{n}\right\}_{n \geq 1}$. Letting $n \uparrow \infty$ in the second inequality, we obtain the inequality concerning the limit functions $\xi$ and $\varphi$ :

$$
|\xi(t)-\xi(s)|^{2} \leq|w(t)-w(s)|^{2}+2 K \sup _{s \leq t_{1} \leq t_{2} \leq t}\left|w\left(t_{2}\right)-w\left(t_{1}\right)\right| .
$$

This implies the continuity of $\xi$. We now prove that $\xi$ is a solution of (2.5) for $0 \leq t \leq T$. For this it is enough to prove that $\varphi$ is associated with $\xi$. First, $\left|\varphi_{n}\right|(T) \leq K$ implies $|\varphi|(T) \leq K$ and hence $\varphi$ is of bounded variation. The condition (2.3) is also verified easily. To verify (2.4), let $\eta \in \mathbf{C}\left(\mathbf{R}_{+}, \bar{D}\right)$ and notice that for $0 \leq t_{1}<t_{2} \leq T$

$$
\begin{aligned}
& \left|\int_{t_{1}}^{t_{2}}\left(\eta(t)-\xi_{n}(t), \varphi_{n}(d t)\right)-\int_{t_{1}}^{t_{2}}(\eta(t)-\xi(t), \varphi(d t))\right| \\
& \quad \leq\left|\int_{t_{1}}^{t_{2}}\left(\xi(t)-\xi_{n}(t), \varphi_{n}(d t)\right)\right|+\left|\int_{t_{1}}^{t_{2}}\left(\eta(t)-\xi(t), \varphi_{n}(d t)-\varphi(d t)\right)\right| .
\end{aligned}
$$

The first is dominated by $K \sup _{t_{1} \leq t \leq t_{2}}\left|\xi(t)-\xi_{n}(t)\right|$ and hence tends to 0 as $n \rightarrow \infty$; the second term also does as can be seen by approximating the integral by the Riemann sum. Therefore

$$
\int_{t_{1}}^{t_{2}}(\eta(t)-\xi(t), \varphi(d t))=\lim _{n \rightarrow \infty} \int_{t_{1}}^{t_{2}}\left(\eta(t)-\xi_{n}(t), \varphi_{n}(d t)\right) \geq 0,
$$

and the proof is finished. 
We proceed to the existence problem for (2.5) assuming that $w \in \mathbf{C}\left(\mathbf{R}_{+}\right.$, $\mathbf{R}^{d}$ ). We begin with the special case when $D$ satisfies the following condition.

Condition $(A)$. There exist a unit vector $\mathrm{e}$ and a constant $c>0$ such that $(e, \boldsymbol{n}) \geq \boldsymbol{c}$ for any $\boldsymbol{n} \in \underset{y \in \partial D}{\cup} \mathcal{N}_{y}(D)$.

Lemma 2.6. Assume that $D$ satisfies the condition $(A)$. Then, there exists a solution $\xi$ of (2.5) for any $w \in \mathbf{C}\left(\mathbf{R}_{+}, \mathbf{R}^{d}\right)$, and for $0 \leq s<t$

$$
\begin{aligned}
& |\xi(t)-\xi(s)| \leq K \Delta_{s, t}, \\
& |\varphi|(t)-|\varphi|(s) \leq K^{\prime} \Delta_{s, t},
\end{aligned}
$$

where $K$ and $K^{\prime}$ are constants depending only upon the constant $c$ in the condition $(A) ; \Delta_{s, t}=\sup _{s \leq t_{1}<t_{2} \leq t}\left|w\left(t_{2}\right)-w\left(t_{1}\right)\right|$.

Proof. For each integer $n \geq 1$ we define $w_{n} \in \mathbf{D}\left(\mathbf{R}_{+}, \mathbf{R}^{d}\right)$ by $w_{n}(t)=w\left(\frac{k}{n}\right)$ for $\frac{k-1}{n} \leq t<\frac{k}{n}, k \geq 1$. Then $w_{n}$ converges to $w$ uniformly on compacts as $n \rightarrow \infty$, and by Lemma 2.1 there exists a solution $\xi_{n}$ of $\xi_{n}=w_{n}+\varphi_{n}$. We put

$$
\begin{aligned}
& \Delta_{n, s, t}=\sup _{s \leq t_{1}<t_{2} \leq t}\left|w_{n}\left(t_{2}\right)-w_{n}\left(t_{1}\right)\right|, \\
& K_{n, s, t}=\left|\varphi_{n}\right|(t)-\left|\varphi_{n}\right|(s),
\end{aligned}
$$

and notice that

$$
\begin{aligned}
\left(\mathfrak{e}, \xi_{n}(t)-\xi_{n}(s)\right) & =\left(\mathfrak{e}, w_{n}(t)-w_{n}(s)\right)+\left(\mathfrak{e}, \varphi_{n}(t)-\varphi_{n}(s)\right) \\
& \geq\left(\mathfrak{e}, w_{n}(t)-w_{n}(s)\right)+c K_{n, \mathbf{s}, t}
\end{aligned}
$$

that is,

$$
K_{n, s, t} \leq\left\{\left|\xi_{n}(t)-\xi_{n}(s)\right|+\Delta_{n, s, t}\right\} / c .
$$

On the other hand, (2.6b) yields

$$
\begin{aligned}
\left|\xi_{n}(t)-\xi_{n}(s)\right|^{2} & \leq \Delta_{n, s, t}^{2}+2 K_{n, s, t} \Delta_{n, s, t} \\
& \leq \Delta_{n, s, t}^{2}+\varepsilon^{2} K_{n, s, t}^{2}+\Delta_{n, s, t}^{2} / \varepsilon^{2}
\end{aligned}
$$

that is,

$$
\left|\xi_{n}(t)-\xi_{n}(s)\right| \leq\left(1+\frac{1}{\varepsilon}\right) \Delta_{n, s, t}+\varepsilon K_{n, s, t}, \quad \varepsilon>0 .
$$

This combined with (2.8) implies

$$
\left|\xi_{n}(t)-\xi_{n}(s)\right| \leq\left(1+\frac{1}{\varepsilon}+\frac{\varepsilon}{c}\right) \Delta_{n, s, t}+\frac{\varepsilon}{c}\left|\xi_{n}(t)-\xi_{n}(s)\right|,
$$


and therefore

$$
\left|\xi_{n}(t)-\xi_{n}(s)\right| \leq K \Delta_{n, s, t}, \quad K_{n, s, t} \leq K^{\prime} \Delta_{n, s, t}
$$

where $K$ is the minimum of $\left(1+\frac{1}{\varepsilon}+\frac{\varepsilon}{c}\right)\left(1-\frac{\varepsilon}{c}\right)^{-1}$ as $\varepsilon$ ranges over the interval $(0, c)$ and $K^{\prime}=(1+K) / c$. In particular, $\left|\varphi_{n}\right|(T)\left(=K_{n, 0, T}\right)$ is bounded and so by Lemma $2.5 \xi_{n}$ converges uniformly on compacts to the solution $\xi$ of (2.5) as $n \rightarrow \infty$. This proves the existence part. The estimates $(2.7 \mathrm{a})$ and $(2.7 \mathrm{~b})$ are also immediate from (2.9). The proof is finished.

Next, we introduce the condition $(B)$ for a convex domain $D$.

Condition $(B)$. There exist $\varepsilon>0$ and $\delta>0$ such that for any $x \in \partial D$ we can find an open ball $B_{\varepsilon}\left(x_{0}\right)=\left\{y \in \mathbf{R}^{d}:\left|y-x_{0}\right|<\varepsilon\right\}$ satisfying $B_{\varepsilon}\left(x_{0}\right) \subset D$ and $\left|x-x_{0}\right|$ $\leq \delta$.

We can easily see that the condition (B) is always satisfied if $D$ is bounded or if $d=2$. We now assume that $D$ satisfies the condition $(B)$ and for a point $x \in \partial D$ put

$$
\begin{aligned}
& B(x)=\left\{y \in \mathbf{R}^{d}:|y-x|<\varepsilon / 2\right\}, \\
& D_{x}=\bigcap_{y \in \partial D \cap \overline{B(x)}} \bigcap_{H \in \mathscr{X}_{y}(D)} H(D),
\end{aligned}
$$

where $H(D)$ denotes the open half space bounded by a supporting hyperplane $H$ and containing $D$. Then $D_{x}$ is a convex domain satisfying the condition $(A)$ with

$$
\mathfrak{e}=\left(x_{0}-x\right) /\left|x_{0}-x\right|, \quad c=\varepsilon / 2 \delta .
$$

Theorem 2.1. (i) Assume that $D$ satisfies the condition (B). Then there exists a unique solution of (2.5) if $w \in \mathbf{C}\left(\mathbf{R}_{+}, \mathbf{R}^{d}\right)$, and the solution depends continuously upon $w$ with respect to the compact uniform topology. (ii) Let $D$ be a general convex domain and $\left\{w_{n}\right\}_{n \geq 1}$ be a sequence in $\mathbf{C}\left(\mathbf{R}_{+}, \mathbf{R}^{d}\right)$ such that $\xi_{n}=w_{n}+\varphi_{n}$ has a solution for each $n$. Assume that $w_{n}$ and $\xi_{n}$ converge to $w$ and $\xi$ uniformly on compacts as $n \rightarrow \infty$, respectively. Then $\xi$ is a solution of (2.5).

Proof. (i) If we put $T_{0}=\inf \{t \geq 0: w(t) \notin \bar{D}\}$, then $\xi_{0}(t) \equiv w(t)\left(0 \leq t \leq T_{0}\right)$ is the solution of (2.5) for $0 \leq t \leq T_{0}$. Assuming that the solution $\xi_{n-1}$ of (2.5) is constructed for $0 \leq t \leq T_{n-1}(n \geq 1)$, we now extend it beyond $T_{n-1}$ as follows. Put $w^{(n)}(t)=w\left(T_{n-1}+t\right), t \geq 0$, let $\xi^{(n)}$ be the solution of $\xi^{(n)}=w^{(n)}+\varphi^{(n)}$ on $\bar{D}_{\xi_{n-1}\left(T_{n-1}\right)}$ and again put

$$
\begin{aligned}
& t_{n}=\inf \left\{t \geq T_{n-1}:\left|\xi^{(n)}\left(t-T_{n-1}\right)-\xi^{(n)}(0)\right|=\varepsilon / 2\right\}, \\
& T_{n}=\inf \left\{t \geq t_{n}: \xi^{(n)}\left(t_{n}-T_{n-1}\right)+w(t)-w\left(t_{n}\right) \notin \bar{D}\right\},
\end{aligned}
$$




$$
\xi_{n}(t)=\left\{\begin{array}{lll}
\xi_{n-1}(t) & 0 \leq t \leq T_{n-1}, \\
\xi^{(n)}\left(t-T_{n-1}\right) & , & T_{n-1} \leq t \leq t_{n}, \\
\xi^{(n)}\left(t_{n}-T_{n-1}\right)+w(t)-w\left(t_{n}\right), & t_{n} \leq t \leq T_{n} .
\end{array}\right.
$$

Then $\xi_{n}$ is the solution of (2.5) on $\bar{D}$ for $0 \leq t \leq T_{n}$. Repeating this argument, we obtain an increasing sequence $\left\{T_{n}\right\}$ and a continuous function $\xi$ defined for $t<T_{\infty}=\lim T_{n}$ such that $\xi$ is the solution of (2.5) for $0 \leq t<T_{\infty}$. The associated function $\varphi$ is flat on each interval $\left(t_{n}, T_{n}\right)$ and (2.7) holds for $s, t \in\left[T_{n-1}, t_{n}\right]$. Therefore, (2.7) holds for $s, t \in\left[T_{n-1}, T_{n}\right]$ with constants $K$ and $K^{\prime}$ depending only on $c=\varepsilon / 2 \delta$. But, (2.7a) with $s=T_{n-1}$ and $t=t_{n}$ implies

$$
\varepsilon / 2 K \leq \Delta_{T_{n-1}, t_{n}} \leq \Delta_{T_{n-1}, T_{n}},
$$

from which we can claim as follows: If $h>0$ is so small that $\Delta_{T}(h)<\varepsilon / 2 K$ where

$$
\Delta_{T}(h)=\max \{|w(t)-w(s)|: 0 \leq s, t \leq T \text { and }|t-s| \leq h\},
$$

$T$ being an arbitrarily fixed positive constant, then $T_{n} \leq T$ implies $T_{n}-T_{n-1}>h$. In other words, $T_{n}>T$ for all $n>T / h$. This fact implies the followings:

(2.10) $T_{n}=\infty$, that is, there exists a solution of (2.5) for $0 \leq t<\infty$.

(2.11) For $0 \leq s<t \leq T$ the solution satisfies

(a) $|\xi(t)-\xi(s)| \leq\left(\frac{T}{h}+1\right) K \Delta_{s, t}$
(b) $|\varphi|(t)-|\varphi|(s) \leq\left(\frac{T}{h}+1\right) K^{\prime} \Delta_{s, t}$

Next, let $\left\{w_{n}\right\}_{n \geq 1}$ be a sequence in $\mathbf{C}\left(\mathbf{R}_{+}, \mathbf{R}^{d}\right)$ converging to $w$ uniformly on compacts and let $\xi_{n}=w_{n}+\varphi_{n}$. Then (b) of (2.11) applied to $\varphi_{n}$ yields

$$
\left|\varphi_{n}\right|(t)-\left|\varphi_{n}\right|(s) \leq\left(\frac{T}{h_{n}}+1\right) K^{\prime} \Delta_{n, s, t}, \quad 0 \leq s<t \leq T .
$$

Here $h_{n}$ depends upon $w_{n}$, but it can be chosen to be independent of $w_{n}$ for all sufficiently large $n$ because $w_{n} \rightarrow w$. Therefore the above inequality on $\left|\varphi_{n}\right|$ implies that $\left\{\left|\varphi_{n}\right|(T)\right\}$ is bounded and hence by Lemma $2.5 \xi_{n} \rightarrow \xi$ uniformly on $[0, T]$.

(ii) Let $T>0$ be any fixed constant. Then there exists $N$ such that

$$
\sup _{n} \max _{0 \leq t \leq T}\left|\xi_{n}(t)\right|<N .
$$

For such an $N$ both $\xi_{n}$ and $\xi$ are the solutions of $\xi_{n}=w_{n}+\varphi_{n}$ and $\xi=w+\varphi, 0 \leq t$ $\leq T$, for the domain $D_{N}=D \cap\{|x|<N\}$. Since $D_{N}$ satisfies the condition $(\dot{B})$, 
we can apply the result of (i) to conclude that $\xi$ is the solution of $(2.5)(0 \leq t \leq T)$ for $D_{N}$ and hence for $D$. The proof is finished.

REMARK 2.3. Even if $D$ does not satisfy the condition $(B)$ (so $d \geq 3$ and $D$ is unbounded), for each $x \in \partial D$ we can find an open ball $B_{\varepsilon}\left(x_{0}\right)=\left\{y \in \mathbf{R}^{d}\right.$ : $\left.\left|y-x_{0}\right|<\varepsilon\right\}$ inside $D$ (but now $\varepsilon$ or $\left|x-x_{0}\right|^{-1}$ is not bounded away from zero as $x$ moves on $\partial D$ ). Therefore, in a manner similar to the proof of Theorem 2.1, (i), we can construct the solution of (2.5) for $t<T_{\infty}=\lim T_{n}$. I can neither prove that $T_{\infty}=\infty$ in general nor give an example in which $T_{\infty}<\infty$.

\section{§3. A stochastic version of (2.5)}

The purpose of this section is to remove the condition $(B)$ in the existence of global solutions of (2.5) by taking $w$ from sample paths of a continuous semimartingale.

Let $(\Omega, \mathscr{F}, P)$ be a complete probability space with an increasing family $\left\{\mathscr{F}_{t}\right\}_{t \geq 0}$ of sub- $\sigma$-fields of $\mathscr{F}$; it is assumed that each $\mathscr{F}_{t}$ contains all $P$-negligible sets and $\mathscr{F}_{t}=\bigcap_{\varepsilon>0} \mathscr{F}_{t+\varepsilon}$. Let $D$ be a convex domain as before.

TheOREM 3.1. Let $\{M(t)\}$ be an $\mathbf{R}^{d}$-valued process with $M(0) \in \bar{D}$ such that each component is a continuous local $\mathscr{F}_{t}$-martingale and $\{A(t)\}$ be an $\mathbf{R}^{d}$ valued, continuous and $\mathscr{F}_{t}$-adapted process of bounded variation with $A(0)=0$.

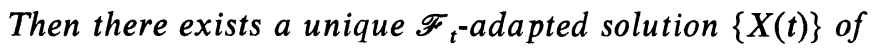

$$
X(t)=M(t)+A(t)+\Phi(t) .
$$

Moreover, for $f \in \mathbf{C}^{2}(\mathbf{R})$ with $f^{\prime} \geq 0$ on $\mathbf{R}_{+}$and $0 \leq s \leq t$ we have

$$
\begin{aligned}
& f\left(|X(t)-X(s)|^{2}\right) \\
& \leq f(0)+2 \int_{s}^{t} f^{\prime} \sum_{i}\left(X^{i}(\tau)-X^{i}(s)\right)\left(M^{i}(d \tau)+A^{i}(d \tau)\right) \\
& \quad+2 \int_{s}^{t} f^{\prime \prime} \sum_{i, j}\left(X^{i}(\tau)-X^{i}(s)\right)\left(X^{j}(\tau)-X^{j}(s)\right) d\left[M^{i}, M^{j}\right] \\
& \quad+\int_{s}^{t} f^{\prime} \sum_{i} d\left[M^{i}, M^{i}\right]
\end{aligned}
$$

where $f^{\prime}, f^{\prime \prime}$ are evaluated at $|X(\tau)-X(s)|^{2}$ and $\left[M^{i}, M^{j}\right]$ denotes the quadratic variation process.

REMARK 3.1. By a solution of (3.1), we mean a $\bar{D}$-valued process $\{X(t)\}$ which satisfies (3.1) almost surely, under the condition that almost all sample paths of $\{\Phi(t)\}$ are associated with those of $\{X(t)\}$. 
Proof. Let $\tau(t)$ be the inverse function of

$$
\theta(t)=t+\sum_{i=1}^{d}\left[M^{i}, M^{i}\right]+|A|(t),
$$

and put

$$
\mathscr{F}_{i}^{*}=\mathscr{F}_{\tau(t)}, M^{*}(t)=M(\tau(t)), \quad A^{*}(t)=A(\tau(t)) .
$$

Then $\left\{M^{*}(t)\right\}$ is a continuous $\mathscr{F}_{t}^{*}$-martingale and $\left\{A^{*}(t)\right\}$ is a continuous $\mathscr{F}_{t}^{*}$ adapted process of bounded variation, satisfying

$$
\begin{aligned}
& 0 \leq \sum_{i, j=1}^{d} x^{i} x^{j} d\left[M^{* i}, M^{* j}\right] \leq|x|^{2} d t, \quad x \in \mathbf{R}^{d}, \\
& A^{*}(t)=\int_{0}^{t} a^{*}(s) d s, \quad\left|a^{*}(t)\right| \leq 1 .
\end{aligned}
$$

Moreover, once we obtain the $\mathscr{F}_{t}^{*}$-adapted solution of $X^{*}(t)=M^{*}(t)+A^{*}(t)$ $+\Phi^{*}(t)$, the $\mathscr{F}_{t}$-adapted solution of (3.1) can be obtained by $X(t)=X^{*}(\theta(t))$. Therefore, in proving the theorem we may assume that $\{M(t)\}$ and $\{A(t)\}$ themselves satisfy (3.3) (without the symbol $*$ ).

First we prove (3.2) assuming the existence of the $\mathscr{F}_{t}$-adapted solution $\{X(t)\}$. An application of Itô's formula yields

$$
\begin{aligned}
f\left(|X(t)-X(s)|^{2}\right)= & \text { the right hand side of (3.2) } \\
& +\int_{s}^{t} f^{\prime} \cdot(X(\tau)-X(s), \Phi(d \tau)) .
\end{aligned}
$$

But the last term is non-positive by $\left(2.4^{\prime}\right)$.

In order to prove the existence of the solution, we first consider the equation for $D_{n}=D \cap\{|x|<n\}$. Taking a point $x^{*}$ in $D_{n}$ (we may consider only those $n$ for which $D_{n} \neq \varnothing$ ), we put

$$
\begin{aligned}
& M_{n}(t)=\mathbf{1}_{D_{n}}(M(0)) M(t)+\mathbf{1}_{\mathbf{R}^{d}-D_{n}}(M(0)) x^{*}, \\
& A_{n}(t)=\mathbf{1}_{D_{n}}(M(0)) A(t) .
\end{aligned}
$$

Since $D_{n}$ is bounded and hence satisfies the condition $(B)$, by Theorem 2.1 there exists a unique $\mathscr{F}_{t}$-adapted solution $\left\{X_{n}(t)\right\}$ of $X_{n}(t)=M_{n}(t)+A_{n}(t)+\Phi_{n}(t)$ for $D_{n}$. If we put

$$
T_{n}=\inf \left\{t \geq 0:\left|X_{n}(t)\right|=n\right\},
$$

then $\left\{X_{n}\left(t \wedge T_{n}\right)\right\}$ is again the solution of $X_{n}\left(t \wedge T_{n}\right)=M_{n}\left(t \wedge T_{n}\right)+A_{n}\left(t \wedge T_{n}\right)+$ $\Phi_{n}\left(t \wedge T_{n}\right)$ for $D_{n}$, and so (3.2) can be applied to $\left|X_{n}\left(t \wedge T_{n}\right)-X_{n}(0)\right|^{2}$. Thus, by taking the expectation we have 


$$
\begin{aligned}
E\left\{\left|X_{n}\left(t \wedge T_{n}\right)-X_{n}(0)\right|^{2}\right\} & \leq 2 \int_{0}^{t} E\left\{\left|X_{n}\left(s \wedge T_{n}\right)-X_{n}(0)\right|\right\} d s+t \\
& \leq \int_{0}^{t} E\left\{\left|X_{n}\left(s \wedge T_{n}\right)-X_{n}(0)\right|^{2}\right\} d s+2 t,
\end{aligned}
$$

and hence

$$
E\left\{\left|X_{n}\left(t \wedge T_{n}\right)-X_{n}(0)\right|^{2}\right\} \leq 2 t e^{t},
$$

that is, $E\left\{\left|X_{n}\left(t \wedge T_{n}\right)\right|^{2}\right\}$ is bounded in $n$ for each fixed $t$. Therefore, for each $t$

$$
P\left\{T_{n} \leq t\right\} \leq E\left\{\left|X_{n}\left(t \wedge T_{n}\right)\right|^{2}\right\} / n^{2} \rightarrow 0 \quad \text { as } n \rightarrow \infty .
$$

On the other hand, the uniqueness lemma in $\S 2$ implies that

$$
T_{n} \leq T_{m} \quad \text { and } \quad X_{n}(t)=X_{m}(t) \quad \text { for } t \leq T_{n}
$$

hold on the set $\left\{M(0) \in \bar{D}_{n}\right\}$ if $n<m$. This fact combined with (3.4) enables us to define $\{X(t)\}$ almost surely by

$$
X(t)=X_{n}(t) \quad \text { on } \quad\left\{M(0) \in \bar{D}_{n}\right\} \cap\left\{t \leq T_{n}\right\} .
$$

Thus defined $\{X(t)\}$ is clearly the $\mathscr{F}_{t}$-adapted solution of (3.1).

\section{$\S 4$. Stochastic differential equation with reflection}

Let $D$ be a convex domain in $\mathbf{R}^{d}$ and $\left\{\Omega, \mathscr{F}, P ; \mathscr{F}_{t}\right\}$ satisfy the same condition as in $\S 3$. We suppose that an $\mathscr{F}_{t}$-adapted $r$-dimensional Brownian motion $B(t)=\left(B^{1}(t), \ldots, B^{r}(t)\right)$ with $B(0)=0$ is given; that is, $\{B(t)\}$ is an $\mathscr{F}_{t}$-adapted continuous process and for $0 \leq s \leq t, \xi \in \mathbf{R}^{d}$

$$
E\left\{e^{i(\xi, B(t)-B(s))} \mid \mathscr{F}_{s}\right\}=e^{-(t-s)|\xi|^{2} / 2}, \text { a.s. }
$$

Given an $\mathbf{R}^{d} \otimes \mathbf{R}^{r}$-valued function $\sigma(t, x)=\left\{\sigma_{k}^{i}(t, x)\right\}$ and an $\mathbf{R}^{d}$-valued function $b(t, x)=\left\{b^{i}(t, x)\right\}$, both being defined on $\mathbf{R}_{+} \times \bar{D}$, we consider the stochastic differential equation with reflection

$$
d X=\sigma(t, X) d B+b(t, X) d t+d \Phi, \quad X(0)=x,
$$

or equivalently

$$
X^{i}(t)=x^{i}+\sum_{k=1}^{r} \int_{0}^{t} \sigma_{k}^{i}(s, X(s)) d B^{k}(s)+\int_{0}^{t} b^{i}(s, X(s)) d s+\Phi^{i}(t),
$$

where $x=\left(x^{1}, \ldots, x^{d}\right) \in \bar{D}$. Our problem is to find an $\mathscr{F}_{t}$-adapted $\bar{D}$-process $\{X(t)\}$ under the condition that $\{\Phi(t)\}$ is an associated process of $\{X(t)\}$. It is always assumed that $\sigma(t, x)$ and $b(t, x)$ are Borel measurable in $(t, x)$. 
THEOREM 4.1. If there exists a constant $K>0$ such that

$$
\begin{aligned}
& \|\sigma(t, x)-\sigma(t, y)\| \leq K|x-y|,|b(t, x)-b(t, y)| \leq K|x-y|, \\
& \|\sigma(t, x)\| \leq K\left(1+|x|^{2}\right)^{1 / 2},\|b(t, x)\| \leq K\left(1+|x|^{2}\right)^{1 / 2},
\end{aligned}
$$

then there exists a (pathwise) unique $\mathscr{F}_{t}$-adapted solution of (4.1) for any $x \in \bar{D}$.

Proof. First we prove the pathwise uniqueness of the solution. Let $\{X(t)\}$ and $\{Y(t)\}$ be $\mathscr{F}_{t}$-adapted solutions of (4.1). Then by the first inequality in Remark 2.2

$$
\begin{aligned}
|X(t)-Y(t)|^{2} \leq & \left|\int_{0}^{t} \sigma(s, X) d B-\int_{0}^{t} \sigma(s, Y) d B\right|^{2} \\
& +2 \int_{0}^{t}(X(s)-Y(s), b(s, X)-b(s, Y)) d s \\
& + \text { the remainder. }
\end{aligned}
$$

Writing the remainder term explicitly, we can see that it has zero expectation ${ }^{1)}$ and hence

$$
\begin{gathered}
E\left\{|X(t)-Y(t)|^{2}\right\} \leq E \int_{0}^{t}\|\sigma(s, X)-\sigma(s, Y)\|^{2} d s \\
+E \int_{0}^{t}|X(s)-Y(s)|^{2} d s+E \int_{0}^{t}|b(s, X)-b(s, Y)|^{2} d s \\
\leq\left(2 K^{2}+1\right) \int_{0}^{t} E\left\{|X(s)-Y(s)|^{2}\right\} d s
\end{gathered}
$$

Therefore, $E\left\{|X(t)-Y(t)|^{2}\right\}=0$.

We give the existence proof, first assuming that $D$ is bounded. By Theorem 2.1 , we can define a sequence $\left\{X^{(n)}(t)\right\}$ of $\bar{D}$-processes by

$$
\begin{aligned}
& X^{(0)}(t)=x \\
& X^{(n)}(t)=x+\int_{0}^{t} \sigma\left(s, X^{(n-1)}\right) d B+\int_{0}^{t} b\left(s, X^{(n-1)}\right) d s+\Phi^{(n)}, n \geq 1 .
\end{aligned}
$$

Then, as in (4.4) we have

1) Because we do not know beforehand that $|X(t)-Y(t)|^{2}$ and the remainder term are integrable, we must employ the following truncation argument. Let $T_{n}$ be the infimum of $t \geq 0$ at which

$$
\int_{0}^{t}|X(s)-Y(s)|^{2} d s+|\Phi|(t)+|\Psi|(t)=n,
$$

and derive (4.4) for $X\left(\cdot \wedge T_{n}\right)$ and $Y\left(\cdot \wedge T_{n}\right)$; it then follows that $E\left\{\left|X\left(t \wedge T_{n}\right)-Y\left(t \wedge T_{n}\right)\right|^{2}\right\}$ $=0$; now let $n \uparrow \infty$. 


$$
E\left\{\left|X^{(n+1)}(t)-X^{(n)}(t)\right|^{2}\right\} \leq\left(2 K^{2}+1\right) \int_{0}^{t} E\left\{\left|X^{(n)}(s)-X^{(n-1)}(s)\right|^{2}\right\} d s .
$$

Therefore, by a routine argument we see that

$$
\int_{0}^{t} \sigma\left(s, X^{(n)}\right) d B+\int_{0}^{t} b\left(s, X^{(n)}\right) d s
$$

is convergent uniformly on compacts as $n \rightarrow \infty$ (a.s.). Consequently, from Theorem 2.1, (i), it follows that $\left\{X^{(n)}(t)\right\}$ is also convergent uniformly on compacts as $n \rightarrow \infty$ (a.s.) and the limit process $\{X(t)\}$ is an $\mathscr{F}_{t}$-adapted solution of (4.1). When $D$ is unbounded, we put $D_{n}=D \cap\{|x|<n\}$ and consider the solution $\left\{X_{n}(t)\right\}$ of (4.1) on $\bar{D}_{n}$. Let $T_{n}$ be the infimum of $t \geq 0$ at which $\left|X_{n}(t)\right|=n$. Then, applying (3.2) to $\left|X_{n}\left(t \wedge T_{n}\right)-x\right|^{2}$ and taking the expectation, we have

$$
\begin{aligned}
E\left\{\mid X_{n}\left(t \wedge T_{n}-\left.x\right|^{2}\right) \leq\right. & 2 E \int_{0}^{t \wedge T_{n}}\left(X_{n}(s)-x, b\left(s, X_{n}\right)\right) d s \\
& +E \int_{0}^{t \wedge T_{n}} \sum_{i, k} \sigma_{k}^{i}\left(s, X_{n}\right)^{2} d s
\end{aligned}
$$

and hence by making use of (4.3)

$$
\begin{aligned}
E\left\{\left|X_{n}\left(t \wedge T_{n}\right)-x\right|^{2}\right\} \leq & \int_{0}^{t} E\left\{\left|X_{n}\left(s \wedge T_{n}\right)-x\right|^{2}\right\} d s \\
& +2 K^{2} \int_{0}^{t} E\left\{1+\left|X_{n}\left(s \wedge T_{n}\right)\right|^{2}\right\} d s
\end{aligned}
$$

Therefore, by Gronwall's inequality we see that $E\left\{\left|X_{n}\left(t \wedge T_{n}\right)\right|^{2}\right\}$ is bounded in $n$ for each fixed $t$ and hence $P\left\{T_{n} \leq t\right\} \leq E\left\{\left|X_{n}\left(t \wedge T_{n}\right)\right|^{2}\right\} / n^{2} \rightarrow 0$ as $n \rightarrow \infty$. On the other hand, by the uniqueness already proved we have $T_{n} \leq T_{m}$ and $X_{n}(t)=X_{m}(t)$ for $t \leq T_{m}$ (a.s.) provided $n<m$, and therefore we can construct an $\mathscr{F}_{t}$-adapted solution $\{X(t)\}$ of (4.1) for $D$ by $X(t)=X_{n}(t), 0 \leq t \leq T_{n}$. This completes the proof.

Theorem 4.2. If $\sigma(t, x)$ and $b(t, x)$ are bounded continuous on $\mathbf{R}_{+} \times \bar{D}$, then on some probability space $(\Omega, \mathscr{F}, P)$ we can find an r-dimensional Brownian motion $\{B(t)\}$ in such a way that (4.1) has a solution.

Proof. We choose sequences $\left\{\sigma_{n}(t, x)\right\}$ and $\left\{b_{n}(t, x)\right\}$ such that (i) $\sigma_{n} \rightarrow \sigma$ and $b_{n} \rightarrow b$ boundedly and uniformly on compacts as $n \rightarrow \infty$, and (ii) $\sigma_{n}$ and $b_{n}$ satisfy the Lipschitz condition. Then there exists a solution $\left\{X_{n}(t)\right\}$ of (4.1) with coefficients $\sigma_{n}$ and $b_{n}$, for each $n$. Making use of (3.2) with $f(u)=u^{p}, p \geq 1$,

$$
\begin{aligned}
E\left\{\left|X_{n}(t)-X_{n}(s)\right|^{2 p}\right\} \leq & c_{p}\left\{E \int_{s}^{t}\left|X_{n}(\tau)-X_{n}(s)\right|^{2 p-1} d \tau\right. \\
& \left.+E \int_{s}^{t}\left|X_{n}(\tau)-X_{n}(s)\right|^{2 p-2} d \tau\right\},
\end{aligned}
$$


where $c_{p}$ is some constant depending on $p$ but not on $n$. Therefore $E\left\{\mid X_{n}(t)\right.$ $\left.-\left.X_{n}(s)\right|^{4}\right\} \leq c|t-s|^{2}$ for $0 \leq s, t \leq T$, where $c$ is some constant depending on $T$ but not on $n, T$ being an arbitrary positive constant. This implies that the family of probability measures on $\mathbf{C}\left(\mathbf{R}_{+}, \bar{D}\right) \times \mathbf{C}\left(\mathbf{R}_{+}, \mathbf{R}^{d}\right)$ induced by $\left\{\left(X_{n}(t), B(t)\right)\right\}$ is tight. Therefore, noticing the result (ii) of Theorem 2.1 we can fill the rest of our proof exactly in the same way as in the case of the whole space $\mathbf{R}^{d}$ (see [5]). The proof is finished.

The following theorem, due to O. Tanbara (unpublished), can be proved in the same way as in [9] making use of the estimate (3.2).

THEOREM 4.3. Let $\rho$ and $\bar{\rho}$ satisfy

$$
\begin{aligned}
& \int_{0+}\left\{\rho^{2}(u) u^{-1}+\bar{\rho}(u)\right\}^{-1} d u=\infty, \\
& \rho^{2}(u) u^{-1}+\bar{\rho}(u) \text { is concave. }
\end{aligned}
$$

Then, for any $\sigma(t, x)$ and $b(t, x)$ satisfying

$$
\|\sigma(t, x)-\sigma(t, y)\| \leq \rho(|x-y|),|b(t, x)-b(t, y)| \leq \bar{\rho}(|x-y|),
$$

the pathwise uniqueness of solutions holds for (4.1).

\section{References}

[1] N. Ikeda, On the construction of two-dimensional diffusion processes satisfying Wentzell's boundary conditions and its application to boundary value problems, Mem. Coll. Sci. Univ. Kyôto, 33 (1961), 367-427.

[2] H. P. McKean, A. Skorohod's integral equation for a reflecting barrier diffusion, J. Math. Kyôto Univ., 3 (1963), 86-88.

[3] H. P. McKean, Stochastic Integrals, Academic Press, 1969.

[4] A. V. Skorohod, Stochastic equations for diffusion processes in a bounded region 1, 2, Theor. Veroyatnost. i Primenen. 6 (1961), 264-274; 7 (1962), 3-23.

[5] A. V. Skorohod, Studies in the Theory of Random Processes, Addison Wesley, 1965.

[6] D. W. Stroock and S. R. S. Varadhan, Diffusion processes with boundary conditions, Comm. Pure Appl. Math., 24 (1971), 147-225.

[7] M. Tsuchiya, On the stochastic differential equation for a two-dimensional Brownian motion with boundary condition, to appear.

[8] S. Watanabe, On stochastic differential equations for multi-dimensional diffusion processes with boundary conditions, J. Math Kyôto Univ., 11 (1971), 169-180.

[9] S. Watanabe and T. Yamada, On the uniqueness of solutions of stochastic differential equations II, J. Math. Kyôto Univ., 11 (1971), 553-563.

Department of Mathematics,

Faculty of Science, Hiroshima University 
